\title{
A flexible electron interferometer demonstrating live phase imaging and interaction- free measurements
}

\author{
Amy Turner ${ }^{1}$, Cameron Johnson ${ }^{2}$ and Benjamin McMorran ${ }^{1}$ \\ ${ }^{1}$ University of Oregon, United States, ${ }^{2}$ University of Oregon, EUGENE, Oregon, United States
}

The resolution for imaging beam-sensitive materials, such as biomolecules, is limited due to highenergy electrons bombarding the sample, precluding imaging cellular dynamics or proteomics at the atomic scale. Currently, the prevailing technique for minimizing damage is to disperse the electron dose over thousands of identical copies of the sample, which requires rigorous computational processing and meticulous sample preparation. Imaging individual radiation-sensitive samples at atomic resolution with negligible damage has been a long-standing goal in electron microscopy, which necessitates a significant dose decrease from current methods. Exploiting quantum protocols is one possible avenue for achieving damage-free imaging. The Elitzur and Vaidman quantum bomb detector ${ }^{1}$ is one proposed mechanism, involving electron interaction-free measurements (IFM) ${ }^{2-4}$.

Here we discuss a novel scanning two-grating Mach-Zehnder interferometer (2GMZI) constructed in a conventional transmission electron microscope (TEM). To demonstrate the capabilities of the 2GMZI, we use it to (1) efficiently make IFMs of an amplitude blocking sample using free electrons ${ }^{5}$ and (2) achieve quantitative phase imaging of a latex nanoparticle. With incremental improvements, the 2GMZI will soon combine direct phase imaging and IFMs to realize interaction-free imaging with electrons.

The Elitzur and Vaidman thought experiment involves a perfectly aligned interferometer, in which a single input quantum traverses the interferometer, self-interferes and is always detected at a single output: the bright port (Fig. 1(a)). However, when an absorbing sample is inserted into one of the interferometer paths, the propagating wavefunction is disrupted causing the dark detector (the dark port) to become viable (Fig. 1(b)). This increase in dark port events is the signature of IFMs, the sample is detected without any interaction from the probing quantum.

We construct the 2GMZI by inserting two nanofabricated diffraction grating holograms ${ }^{6}$ into the TEM column, functionally used as beam splitters (Fig. $1(\mathrm{a}, \mathrm{b})$ ). The first grating provides two predominant paths which spatially separate, interact with the sample, recombine and co-propagate to the output detector. To achieve the proper IFM alignment of our 2GMZI, we shift the relative phase between the two gratings (Fig. 2(b)) such that the co-propagating output probe destructively interferes and is denoted as the dark port (Fig. 2(a)). To demonstrate an IFM with free electrons, ${ }^{5}$ we insert an opaque knife-edge (an aperture edge) to block one of the interferometer paths. Using the CCD we select regions of the camera as the bright and dark ports. The 2GMZI achieved an IFM efficiency ${ }^{5}$ of $\eta=20.7 \pm 2.3 \%$. Incomplete suppression of the dark port when the object was removed resulted in an $8.7 \pm 0.7 \%$ probability for false positives.

Furthermore, we employ the scan coils to raster the probes across the sample and demonstrate direct phase imaging of a $50 \mathrm{~nm}$ latex nanoparticle on graphene. In our current 2GMZI, the intensity in the minimized dark port is non-negligible, inhibiting IFM imaging. However, we are able to gain live, quantitative phase information with the scanning 2GMZI. As seen in Fig. 2(c,d), we can align the dark port to the maximum or minimum, scan over the sample and directly image the imparted phase from a sample. We recover the phase with a resolution of $\sigma_{\mathrm{ph}}=200 \mathrm{mrad}$. Current work is focused on decoupling phase and amplitude loss, improving the spatial resolution, and enhancing the 2GMZI components to achieve stable IFM alignment while scanning.

This 2GMZI is particularly flexible and effectual due to the tunable path separation, ability to arbitrarily apply phase shifts between paths by adjusting the relative grating positions, accessibility of individual paths in the sample plane, scanning capabilities for imaging, and the ability to gain real time 
phase information, all the while using a conventional bright field detector. Here we employed the 2GMZI to demonstrate IFMs with free electrons and direct phase imaging. When achieved simultaneously, interaction-free imaging will improve low-dose phase imaging of radiation-sensitive samples, decreasing the required dose for high-resolution imaging to well below the damage threshold.

(a)

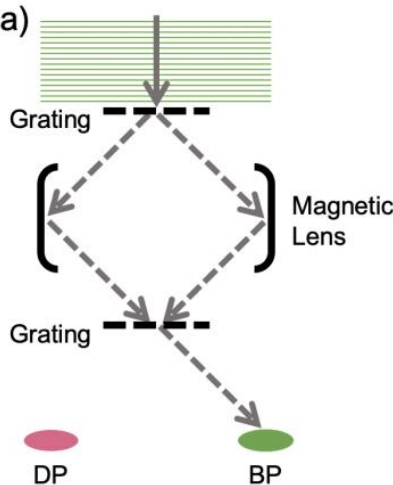

(b)

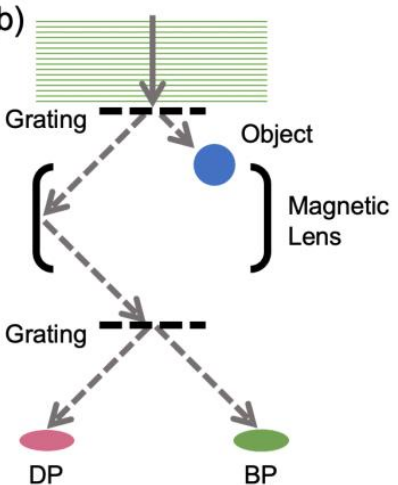

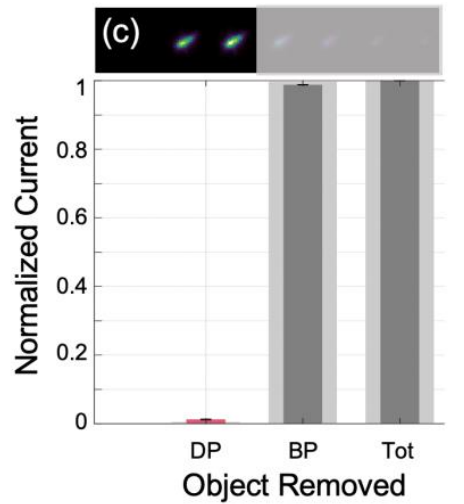

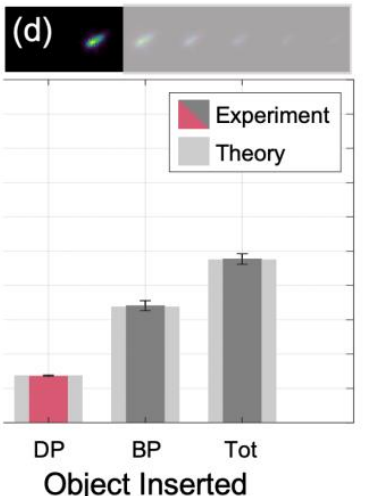

Figure 1. Schematic of the Elitzur and Vaidman thought experiment in an electron microscope employing two gratings with an opaque object removed (a) and inserted (b). The experimental total (Tot), bright port (BP) and dark port (DP) currents when an opaque object is removed (c) and inserted (d), normalized to the total current when the object is removed.
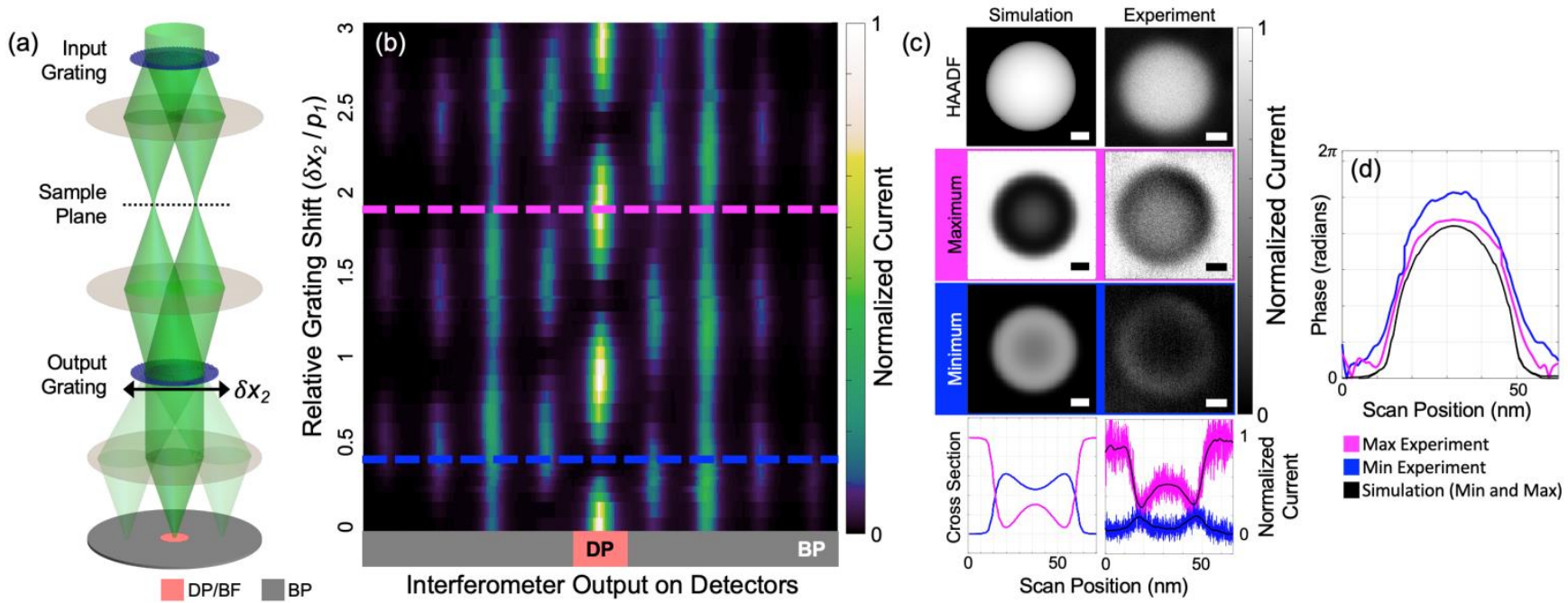

Figure 2. (a) Schematic of the two-grating Mach-Zehnder interferometer (2GMZI) with the recombined diffraction order (the dark port or DP) focused on the bright field (BF) detector. (b) Normalized output currents of the 2GMZI for different relative grating shifts, $\delta \times 2 / \mathrm{p} 1$ (position of the second grating over the input grating pitch). (c) Simulated and experimental HAADF and interferometric images of a latex particle on graphene, aligned both at the maximum (magenta) and minimum (blue). The cross sections of the interferometric images are displayed in the bottom row, both raw and smoothed (black). (d) Recovered phase from the interferometer output. Scalebars represent $10 \mathrm{~nm}$.

References

1. Elitzur, A. C. \& Vaidman, L. Quantum mechanical interaction-free measurements. Found Phys 23, 987-997 (1993).

2. Putnam, W. P. \& Yanik, M. F. Noninvasive electron microscopy with interaction-free quantum measurements. Phys. Rev. A 80, 040902 (2009). 
3. Kruit, P. et al. Designs for a quantum electron microscope. Ultramicroscopy 164, 31-45 (2016).

4. Juffmann, T. et al. Multi-pass transmission electron microscopy. Sci Rep 7, 1699 (2017).

5. Turner, A. E., Johnson, C. W., Kruit, P. \& McMorran, B. J. Interaction-free measurement with electrons. Under Review (2021).

6. Johnson, C. W. et al. Exact design of complex amplitude holograms for producing arbitrary scalar fields. Opt. Express 28, 17334 (2020).

7. Kwiat, P., Weinfurter, H., Herzog, T., Zeilinger, A. \& Kasevich, M. A. Interaction-Free Measurement. Phys. Rev. Lett. 74, 4763-4766 (1995). 